\title{
Suplementação de creatina: efeitos ergogênicos e terapêuticos
}

\author{
Creatine supplementation: ergogenic and \\ therapeutic effects
}

\author{
Lucas Caseri Câmara1, Raphael Mendes Ritti Dias²
}

\begin{abstract}
Câmara LC, Dias RMR. Suplementação de creatina: efeitos ergogênicos e terapêuticos. Rev Med (São Paulo). 2009 abr.-jun.;88(2):94-102.

RESUMO: A creatina foi descoberta há mais de um século, porém seu uso tornou-se importante no cenário esportivo a partir dos anos 70. Desde então, o aprofundamento dos conhecimentos e dos protocolos de utilização fez desta substância o recurso ergogênico lícito mais estudado e utilizado por atletas atualmente. Os benefícios da suplementação em atletas serviram de modelo para estudo da utilização como adjuvante terapêutico em indivíduos doentes, que apresentam alguma deficiência de síntese e ressíntese de ATP, doenças neurológicas e musculares, ou que cursam com atrofia ou prejuízo do metabolismo muscular.
\end{abstract}

DESCRITORES: Creatina. Trifosfato de adenosina. Suplementação alimentar.

\section{INTRODUÇÃO}

A creatina tem sido um suplemento nutricional amplamente utilizado nos últimos anos, sobretudo por atletas, devido aos seus possíveis efeitos ergogênicos sobre a massa muscular e o desempenho físico.

De fato, existe grande corpo de evidências na literatura indicando que a suplementação de creatina pode potencializar alterações na composição corpo- ral, principalmente no tecido muscular, bem como na realização de esforços físicos predominantemente anaeróbios.

Mais recentemente, a creatina passou a ser estudada em indivíduos portadores de doenças crônicas não transmissíveis, e os resultados têm indicado um possível efeito terapêutico com a suplementação dessa substância, o que sugeriria sua utilização no tratamento dos pacientes. Em contrapartida, a literatura também tem trazido

\footnotetext{
1. Médico, especializado em Fisiologia do Exercício (CECAFI-FMUSP / CEFE-UNIFESP). Mestrando em Ciências - Fisiopatologia Experimental - FMUSP.

2. Educador Físico, doutor em Saúde Pública (FSP-USP). Docente da Escola Superior de Educação Física da Universidade de Pernambuco.

Endereço para correspondência: Lucas Caseri Câmara. Rua Alexandre Herculano, 115, Boqueirão, Santos, SP. CEP: 11050-131. E-mail: Iccamara@usp.br
} 
informações sobre os possíveis efeitos colaterais da suplementação de creatina, o que, por sua vez, pode ser considerado um risco para a saúde.

Nesse sentido, o objetivo deste estudo é revisar a literatura referente aos efeitos da suplementação de creatina, descrevendo temas atuais referentes à utilização dessa substância. Assim, serão apresentados: um breve relato histórico da utilização da creatina; as características de síntese, absorção e transporte; os efeitos ergogênicos; seus possíveis efeitos terapêuticos; e, finalmente, seus possíveis efeitos colaterais.

\section{Histórico}

A creatina (ácido acético $\alpha$-metil guanidina), uma substância orgânica encontrada na carne vermelha foi descoberta pelo cientista francês Michel Eugene Chevreul em 1832 (Figura 1) ${ }^{35,85}$. Passados quinze anos (1847), Justus Von Liebg, observou que animais selvagens (raposas) apresentavam aproximadamente dez vezes mais conteúdo de creatina em sua musculatura quando comparadas a animais domesticados, suspeitando que de alguma forma esta substância exercesse influência na atividade muscular, visto que animais selvagens possuíam maior capacidade e velocidade de locomoção ${ }^{35,85}$.

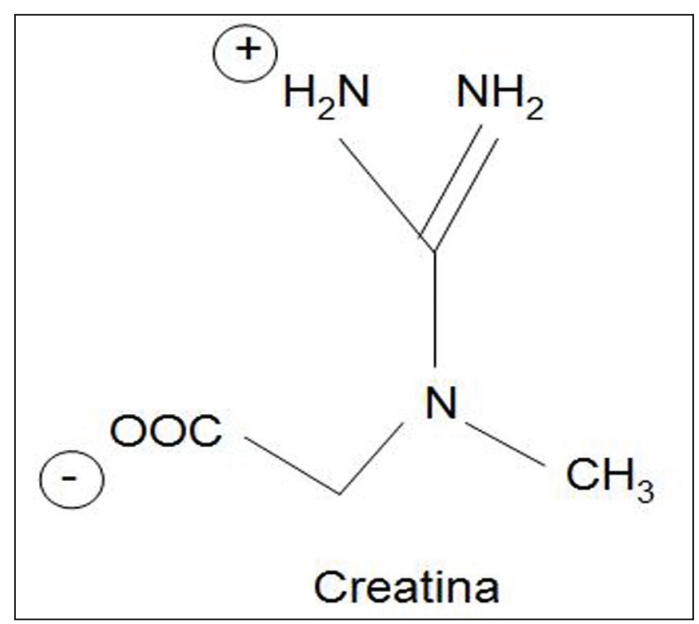

FIGURA 1. Ácido acético $\alpha$-metil guanidina

Em 1880, o atual metabólito conhecido da degradação da creatina, a creatinina, foi isolada em amostras de urina ${ }^{85}$. A participação da creatina como componente do metabolismo energético muscular foi confirmada em 1927, e, mais especificamente, que a CK (creatina quinase) era enzima chave no processo de fosforilação da creatina em $1934^{19}$.

Os primeiros estudos realizados com biópsia por agulha foram realizados em 1968, detectando que a creatina também estava presente na musculatura esquelética de humanos ${ }^{20}$. Nos anos subsequentes (1970-80), a utilização da creatina como potencial artifício de melhoria do desempenho de atletas começou a ser aventada e testada ${ }^{86}$.

Os primeiros relatos de que medalhistas olímpicos estariam utilizando creatina vieram das Olimpíadas de 1992, e, nos Jogos Olímpicos seguintes, a utilização da substância era estimada em mais de $80 \%$ dos atletas ${ }^{85,86}$. Atualmente a creatina monohidratada pode ser colocada entre os melhores recursos ergogênicos lícitos, utilizado por atletas de alto rendimento ${ }^{8}$.

Fonte, síntese, armazenamento e metabolismo

A creatina é uma amina nitrogenada, que pode ser encontrada naturalmente em maiores concentrações nas carnes (vaca e porco) e nos peixes (atum, arenque, e salmão) ${ }^{4}$ (Tabela 1). Em dietas balanceadas utilizadas por indivíduos fisicamente ativos (1-2g de proteína por kilo de peso corporal), podem ser conseguidos aproximadamente $50 \%$ das necessidades de creatina, de forma que o restante é endogenamente sintetizado pelos rins, fígado e pâncreas, através dos aminoácidos glicina, arginina e metionina ${ }^{88}$. Mais de $90 \%$ da creatina em humanos é encontrada na musculatura esquelética, e sua principal função fisiológica é fornecer energia rápida para esforços curtos e intensos, através da formação da fosfocretina e da ressíntese do ATP ${ }^{53}$. O restante se distribui na musculatura lisa (coração), nas células sanguíneas (série branca e vermelha), nos testículos (espermatozóides), no cérebro e na retina $^{88}$ (Figura 2).

TABELA 1. Quantidade de Creatina em alimentos selecionados

\begin{tabular}{lc}
\hline Alimento & Quantidade de Creatina $(\mathrm{g} / \mathrm{kg})$ \\
\hline Bacalhau & 3,0 \\
\hline Atum & 4,0 \\
Salmão & 4,5 \\
\hline Arenque & $6,5-10,0$ \\
Carne de vaca & 4,5 \\
Carne de porco & 5,0 \\
Leite de vaca & 0,1 \\
\hline
\end{tabular}

Adaptado de Balsom et al. ${ }^{4}$

Para um homem de 70 quilos, a concentração máxima encontrada na musculatura esquelética fica em torno de $160 \mathrm{Mmol} / \mathrm{kg}$ (aproximadamente 120g). Todavia, os níveis de creatina são influenciados por 
diversos fatores, dentre os quais estão idade, nível de condicionamento físico, tipo de fibra muscular predominante, concentração sérica de hormônios (insulina, IGF-1, catecolaminas, e hormônio tireóideo - T3) e doenças associadas ${ }^{29,69,72}$.

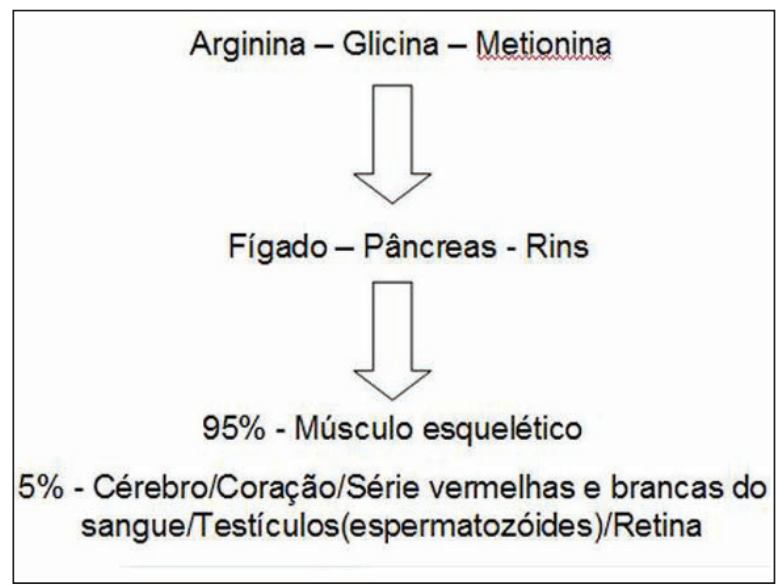

FIGURA 2. Síntese e armazenamento da creatina

A creatina encontra-se na musculatura esquelética principalmente $(65 \%)$ sob a forma de creatina fosfato (CP), e o restante (35\%), na forma de creatina livre. Juntas, essas duas reservas constituem a fonte de geração de energia do Sistema P-Cr (creatinafosfato) $^{42}$ (Figura 3). Este sistema pode gerar grandes quantidades de energia em um curto espaço de tempo (alta potência), porém, a duração desta energia gerada é muito curta ${ }^{52}$ (Figura 4). O catabolismo de PCR é capaz de gerar de modo instantâneo aproximadamente 9 mmol de ATP por quilo de peso corporal por segundo, superando em mais de mil vezes a capacidade de geração de energia fornecida pelas vias aeróbias no mesmo período de tempo ${ }^{52}$.

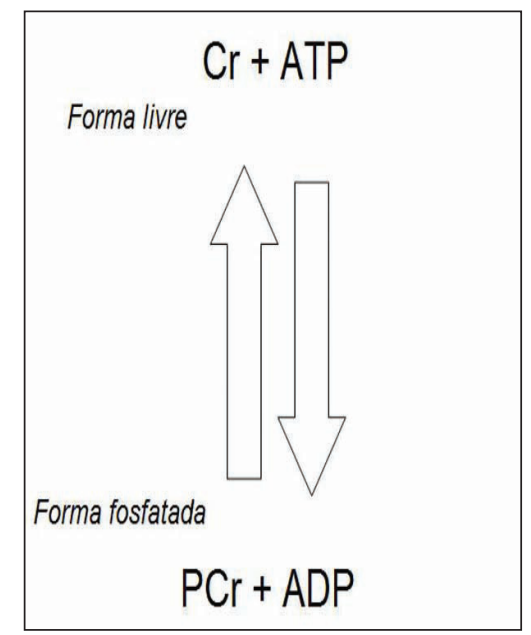

FIGURA 3. Creatina Livre e Creatina Fosfato (ATP: adenosina trifosfato; ADP: adenosina difosfato)

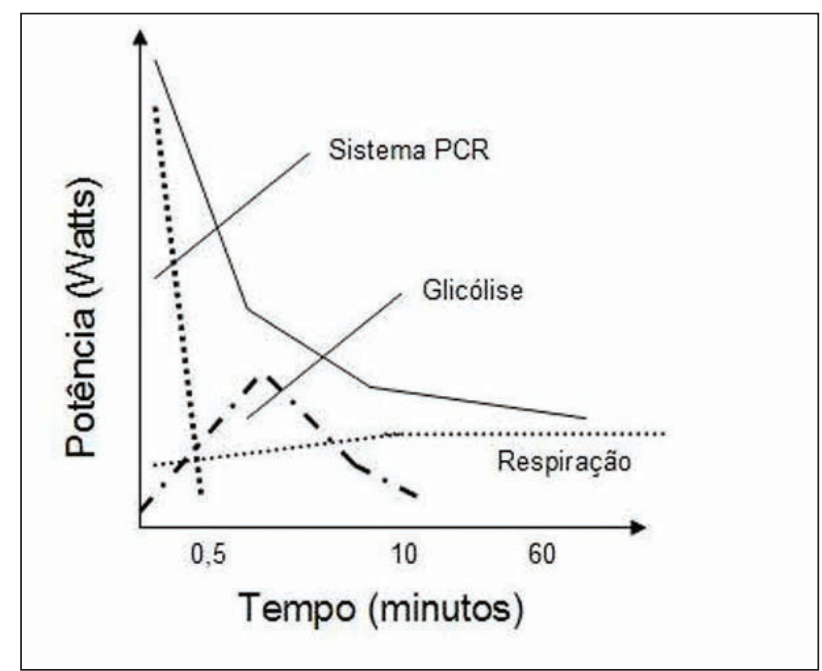

FIGURA 4. Sistema PCR (fosfocreatina) de geração de energia para o metabolismo

\section{Efeitos ergogênicos}

Segundo Williams et al. ${ }^{85}$, os principais efeitos ergogênicos relacionados à suplementação oral de creatina monohidratada, e o conseqüente aumento das concentrações intramusculares da substância são: 1) Aumento da disponibilidade de CP (creatina fosfato) aumentando a quantidade potencial de energia disponível; 2) Aumento da taxa de ressíntese de ATP, prolongando o tempo de geração de energia rápida; 3) Redução da acidez muscular, e conseqüente retardo do aparecimento da fadiga; 4) Aumento da massa e força muscular, proporcionando aumento no nível de treinamento atingido.

Estudos de revisão ${ }^{36,49,84}$ e meta-análise $e^{9,21}$ confirmam a melhora do desempenho esportivo, da composição corporal (aumento de massa muscular), da força, potência e resistência, além de permitir melhor desempenho em sessões de treinamentos máximos (exaustivos e repetitivos).

Segundo Kreider et al. ${ }^{42}$, que revisaram mais de 300 estudos sobre o tema, afirmam que em $70 \%$ deles os efeitos foram positivos e promoveram melhora do desempenho esportivo. Os resultados indicaram aumentos médios variando entre 5-15\% na capacidade de potência máxima, força e velocidade; 1-5\% no trabalho realizado em contrações máximas; $5-15 \%$ no desempenho de sprints repetitivos; e aumento de 1-2 kg de massa magra. Muito embora as alterações possam parecer pequenas, os autores ressaltam que os indivíduos estudados eram atletas, em que pequenas alterações nos componentes da aptidão física podem ser altamente significantes nos resultados esportivos ${ }^{42}$. 
Williams et al. ${ }^{85}$ sugerem haver uma possível melhora do desempenho também em atividades prolongadas, devido à melhora do metabolismo oxidativo (aumento da atividade da enzima citrato sintase e aumento da taxa de ressintese aeróbia de ATP). No entanto, estudos mais recentes têm refutado essa hipótese ${ }^{60,81}$. Além disso, esses estudos também têm sugerido que o aumento de massa magra, grande parte em virtude do aumento da hidratação muscular, poderia ter efeito prejudicial nas atividades esportivas em que não é desejável este aumento (ex. provas de longa duração).

A Sociedade Internacional de Nutrição Esportiva (International Society of Sports Nutrition), publicou recentemente um posicionamento sobre a suplementação de creatina, e, neste documento, os autores afirmam que a substância é atualmente o suplemento mais estudado e clinicamente mais significativo para aumentar o volume muscular e a capacidade de exercício em esforços de altas intensidades em atletas ${ }^{8}$.

\section{Protocolo de utilização}

O protocolo de suplementação sugerido como mais adequado, utiliza uma fase de saturação $(0,3 g /$ $\mathrm{kg} / \mathrm{dia}$, por 3 a 5 dias, divididos em 4-5 doses por dia, adicionados de carboidratos), e uma fase de manutenção (3-5g/dia, podendo ser feita em uma única dose $)^{8}$. Alguns autores ${ }^{53,85}$ afirmam que não é necessário fazer interrupções ou pausas durante a utilização continuada, pois apesar da síntese endógena ficar inibida com uso crônico, esta função se restabelece, normalmente, em 4 semanas.

\section{Efeitos terapêuticos}

A creatina participa ativamente do metabolismo cerebral, sendo essencial para este ${ }^{1}$. O armazenamento cerebral da substância está nos locais de alta necessidade energética, fornecendo energia através da ressíntese de ATP para os neurônios ${ }^{1}$. Síndromes ao nascimento que cursam com deficiência de creatina acabam por prejudicar o desenvolvimento do sistema nervoso e consequentemente o desempenho intelectual|3,70,71. Nestes casos, tem-se utilizado a suplementação de creatina, com melhoras deste desempenho, além de também ser a creatina responsável pela promoção de diferenciação celular de precursores de neurônios (fato que possivelmente contribui para regeneração neuronal em casos de lesão celular) ${ }^{3,7,59,70,71}$. A deficiência de ingesta protéica na infância prejudica o desenvolvimento e capacidade intelectual ${ }^{1,71}$. Populações que têm baixa ingesta protéica (por exemplo, os idosos), que não obtém da dieta níveis adequados de creatina podem se beneficiar dessa suplementação, obtendo melhoras do desempenho intelectual ${ }^{46,59}$.

Estudos têm avaliado o potencial da creatina como adjuvante no tratamento ${ }^{6}$ ou na prevenção de doenças ${ }^{89}$, e sua melhor contribuição ocorre quando atrofias musculares estão associadas, ou quando a síntese de creatina ou a ressíntese de ATP se apresentam de alguma forma prejudicadas. A utilização da suplementação de creatina com funções terapêuticas já foi pesquisada em diversas doenças com alterações neurológicas e musculares (distrofias e alterações neuromusculares ${ }^{39,40,51,77}$, Parkinson ${ }^{32}$, doença de Hungtinton ${ }^{33,45}$, miopatias inflamatórias ${ }^{18,38}$, doença de Charcot Marie Tooth ${ }^{68}$, esclerose lateral amiotrófica ${ }^{24}$, isquemia cerebral e trauma crânio encefálico ${ }^{63,87}$, esclerose múltipla ${ }^{37}$, Alzheimer $^{75}$, miopatia induzida por corticoesteróides ${ }^{47}$, distrofia de Duchenne ${ }^{76}$, citopatia mitocondrial ${ }^{78}$ ), em doenças que cursam com erros do metabolismo (doença de McArdle ${ }^{58}$ e Atrofia girata ${ }^{67,82}$ ), em imobilizações ${ }^{66}$ e pós-operatórios ortopédicos ${ }^{54,64}$, em doenças respiratórias ${ }^{27}$ e cardiovasculares ${ }^{2,26,28,34}$. Os resultados destes estudos têm evidenciado que a suplementação de creatina como adjuvante terapêutico, além de segura (sem evidências de efeitos colaterais), proporcionou melhorias do metabolismo e da qualidade muscular, contribuindo para a melhora da aptidão física associado à redução da sarcopenia ${ }^{2,18,24,26-28,32-34,37-40,45,47,51,54,58,63,64,66-68,75-78,82,87}$.

Indivíduos idosos frequentemente apresentam redução da força, da resistência e da massa muscular, contribuindo para a menor aptidão física observada nesta população ${ }^{23,44}$. Como adjuvante terapêutico para reduzir estas adversidades decorrentes do envelhecimento sedentário, o treinamento resistido tem sido recomendado como intervenção segura e eficaz ${ }^{11}$. A literatura apresenta estudos sobre o fato de que os benefícios de aumento da força, resistência, massa muscular e aptidão física conseguidos com esta modalidade ${ }^{22,25}$ podem ser potencializados quando a suplementação de creatina é associada13,14,75.

Chilibeck et al. ${ }^{16}$ observaram em idosos o aumento significante da densidade mineral óssea, da força e da massa muscular, em apenas três meses de suplementação associada ao treinamento resistido, quando comparado ao grupo placebo.

Brose et al. ${ }^{10}$ em estudo controlado, observaram que, após 14 semanas de associação de treinamento resistido e suplementação de creatina em idosos, houve um aumento significante na capacidade de realização de tarefas funcionais (sentar e levantar da cadeira, tempo de caminhada, e capacidade de subir escadas), e também da força e da massa muscular.

A associação de treinamento e suplementação, 
segundo Chrusch et al. ${ }^{17}$, além de melhorar a força e composição corporal, aumenta também a resistência muscular.

Os efeitos da utilização de creatina e treinamento resistido em idosos, quando associado também à suplementação protéica, pode incrementar ainda mais os ganhos na força e massa muscular ${ }^{15}$.

\section{Efeitos colaterais e função renal}

A suplementação de creatina quando feita segundo as recomendações citadas parece não trazer efeitos colaterais ${ }^{8}$.

Importantes entidades como a US Food and Drog Administration (FDA), a Association of Professional Team Physicians, a International Society of Sports Nutrition e o American College of Sports Medicine (ACSM) atestam a segurança da suplementação de creatina ${ }^{8,79}$.

No entanto, sabe-se que altas doses (acima de $10 \mathrm{~g} /$ dose) de creatina, tomadas de uma única vez, podem provocar náuseas, vômitos, diarréia, cefaléia e mal estar geral, sem evidências de efeitos colaterais mais graves ${ }^{50}$

Existem diversos relatos de caso na literatura que apontam para potenciais efeitos nefrotóxicos ou letais da creatina ${ }^{5,41,43,56,57,61,80}$. No entanto, tais publicações são estudos de caso retrospectivos, o que per se não permitiriam as tais generalizações feitas pelos autores ${ }^{31}$. Somado a isso, temos também que estes estudos apresentam falhas metodológicas na avaliação da função renal com marcadores não adequados para o propósito, além do pouco detalhamento sobre as comorbidades e histórico clínico dos pacientes ${ }^{31}$. Tais estudos são devidamente considerados pelas entidades esportivas em seus posicionamentos mais atuais, no entanto, são relevados segundo a sua importância ${ }^{8,79}$.

Estudos realizados em indivíduos com deficiência da síntese de creatina não apontaram efeitos colaterais em períodos prolongados de utilização ${ }^{67,82}$. Já estudos com outras populações sugerem que a suplementação de creatina não prejudica a função renal ${ }^{30,55,73,74,90}$, hepática ${ }^{83}$ e do sistema cardiovascular ${ }^{48}$, bem como inúmeros marcadores de saúde ${ }^{12,62,65}$.

Gualano et al. ${ }^{30}$ realizaram recentemente um elegante estudo randomizado e controlado avaliando a segurança da suplementação de creatina sobre a função renal. Segundo os autores, foi o primeiro estudo que utilizou como marcador da função renal a Cistatina $\mathrm{C}$, tido como melhor marcador para este propósito, uma vez que não sofre influência da quantidade protéica dietética ${ }^{30}$. Outra vantagem da utilização deste marcador (Cistatina $\mathrm{C}$ ) é que a creatina sofre conversão espontânea $(\sim 1,7 \%$ ao dia) para creatinina ${ }^{53,88}$, aumentando estes valores em uma série de estudos prévios, questionando a validade da utilização da creatinina como marcador de piora da função renal observada nestes estudos.

É importante que a maioria dos estudos que utilizaram a creatina em pacientes com doenças crônicas, analisaram os efeitos dessa substância na função renal por curtos períodos. Nesse sentido, ainda não existem evidências que permitam fundamentar a segurança da utilização dessa substância em períodos prolongados.

\section{CONCLUSÕES}

A grande maioria das evidências disponíveis na literatura sugere que a suplementação de creatina monohidratada, quando realizada dentro do protocolo sugerido, parece ser excelente recurso ergogênico. Com relação aos efeitos terapêuticos da creatina, os estudos sugerem que a suplementação pode ser considerada um potencial adjuvante terapêutico para diversas doenças. Todavia, vale ressaltar, que a utilização da creatina como ferramenta terapêutica ainda deve ser vista com cautela, uma vez que ainda há poucos estudos, muitos deles com resultados controversos, com limitações metodológicas importantes e que ainda não investigaram o impacto da suplementação dessa substância por um longo período. Além disso, não há estudos avaliando os efeitos da utilização continuada de creatina em indivíduos doentes, que atestem que os benefícios superem os potenciais riscos.

Câmara LC, Dias RMR. Creatine supplementation: ergogenic and therapeutic effects. Rev Med (São Paulo). 2009 abr.-jun.;88(2):94-102.

\begin{abstract}
Creatine was discovered more than one century ago, but its use became important in sports scenario in the 70's. Since then, the improvement in knowledge and utilization protocols made this substance the licit ergogenic aid most studied and used by athletes nowadays. The benefits of supplementation in athletes served as a model for studies in diseased individuals, that present ATP synthesis or ressynthesis deficiency, neurological and muscular diseases, or that suffer from muscular atrophy or impairment of muscular metabolism.
\end{abstract}

KEY WORDS: Creatine. Adenosine triphosphate. Supplementary feeding. 


\section{REFERÊNCIAS}

1. Andres RH, Ducray AD, Schlattner U, Wallimann T, Widmer HR. Functions and effects of creatine in the central nervous system. Brain Res Bull. 2008;76:329-43.

2. Andrews R, Greenhaff P, Curtis S, Perry A, Cowley AJ. The effect of dietary creatine supplementation on skeletal muscle metabolism in congestive heart failure. Eur Heart J. 1998;19:617-22.

3. Arias A, Corbella M, Fons C, Sempere A, Garcia-Villoria J, Ormazabal A, et al. Creatine transporter deficiency: prevalence among patients with mental retardation and pitfalls in metabolite screening. Clin Biochem. 2007;40:1328-31.

4. Balsom P, Söderlund K, Ekblom B. Creatine in humans with special reference to creatine supplementation. Sports Med. 1994;18:268-80.

5. Barisic N, Bernert G, Ipsiroglu O, Stromberger C, Muller T, Gruber S, et al. Effects of oral creatine supplementation in a patient with MELAS phenotype and associated nephropathy. Neuropediatrics. 2002;33:157-61.

6. Benzi G, Ceci A. Creatine as nutritional supplementation and medicinal product. J Sports Med Phys Fitness. 2001;41(1):1-10.

7. Bianchi MC, Tosetti M, Battini R, Leuzzi V, Alessandri MG, Carducci $C$, et al. Treatment monitoring of brain creatine deficiency syndromes: a $1 \mathrm{H}$ - and 31P-MR spectroscopy dtudy. Am J Neuroradiol. 2007;28:548-54.

8. Boford TH, Kreider RB, Stout JR, Greenwood M, Campbell B, Spano M, et al. International Society of Sports Nutrition position stand: creatine supplementation and exercise. J Int Soc Sports Nutr. 2007;4:6.

9. Branch JD. Effect of creatine supplementation on body composition and performance: a meta-analysis. Int $\mathrm{J}$ Sport Nutr Exerc Metab. 2003;13(2):198-226.

10. Brose A, Parise G, Tarnopolsky MA. Creatine supplementation enhances isometric strength and body composition improvements following strength exercise training in older adults. J Gerontol A Biol Sci Med Sci. 2003;58(1):11-9.

11. Câmara LC, Santarém JM, Jacob-Filho W. Atualização dos conhecimentos sobre a prática de exercícios resistidos por indivíduos idosos. Acta Fisiatr. 2008;15(4):257-62.

12. Cancela P, Ohanian C, Cuitiño E, Hackney AC. Creatine supplementation does not affect clinical health markers in soccer players. Br J Sports Med. 2007 [Epub ahead of print].

13. Candow DG, Chilibeck PD Effect of creatine supplementation during resistance training on muscle accretion in the elderly. J Nutr Health Aging. 2007;11(2):185-8.

14. Candow DG, Chilibeck PD. Timing of creatine or protein supplementation and resistance training in the elderly. Appl Physiol Nutr Metab. 2008;33(1):184-90.

15. Candow DG, Little JP, Chilibeck PD, Abeysekara S, Zello GA, Kazachkov M, et al. Low-dose creatine combined with protein during resistance training in older men. Med Sci Sports Exerc. 2008;40(9):1645-52.

16. Chilibeck PD, Chrusch MJ, Chad KE, Davison KS, Burke DG. Creatine monohydrate and resistance training increase bone mineral content and density in older men. J Nutr Health Aging. 2005;9(5):352-5.

17. Chrusch MJ, Chilibeck PD, Chad KE, Davison KS, Burke AG. Creatine supplementation combined with resistance training in older men. Med Sci Sports Exerc. 2001;33(12):2111-7.

18. Chung YL, Alexanderson $\mathrm{H}$, Pipitone N, Morrison C, Dastmalchi $\mathrm{M}$, Ståhl-Hallengren $\mathrm{C}$, et al. Creatine supplements in patients with idiopathic inflammatory myopathies who are clinically weak after conventional pharmacologic treatment: Six-month, double-blind, randomized, placebo-controlled trial. Arthritis Rheum. 2007;57(4):694-702.

19. Conway MA, Clark JF. Creatine and creatine phosphate: scientific and clinical perspectives. Orlando, FL: Academic Press; 1996.

20. Cooper AC, Miller JR. Progressive muscular dystrophy: a review. Rev Can Biol. 1962;21:337-51.

21. Dempsey RL, Mazzone MF, Meurer LN. Does oral creatine supplementation improve strength? A metaanalysis. J Fam Pract. 2002;51(11):945-51.

22. Dias RM, Gurjão AL, Marucci MF. Benefícios do treinamento com pesos para a aptidão física de idosos. Acta Fisiatr. 2006;13(2):90-5.

23. Doherty TJ. Invited review: aging and sarcopenia. J Appl Physiol. 2003;95(4):17217-27.

24. Ellis AC, Rosenfeld J. The role of creatine in the management of amyotrophic lateral sclerosis and other neurodegenerative disorders. CNS Drugs. 2004;18(14):967-80.

25. Faria JC, Machala CC, Dias RC, Dias JMD. Importância do treinamento de força muscular na reabilitação da função muscular, equilíbrio e mobilidade de idosos. Acta Fisiatr. 2003;10(3):133-7.

26. Ferraro S, Codella C, Palumbo F, Desiderio A, Trimigliozzi $P$, Maddalena $G$, et al. Hemodynamic effects of creatine phosphate in patients with congestive heart failure: a double-blind comparison trial versus placebo. Clin Cardiol. 1996;19:699-703.

27. Fuld JP, Kilduff LP, Neder JA, Pitsiladis Y, Lean ME, Ward SA, Cotton MM. Creatine supplementation during pulmonary rehabilitation in chronic obstructive pulmonary disease. Thorax. 2005;60(7):531-7.

28. Gordon A, Hultman E, Kaijser L, Kristjansson S, Rolf 
CJ, Nyquist $\mathrm{O}$, et al. Creatine supplementation in chronic heart failure increases skeletal muscle creatine phosphate and muscle performance. Cardiovasc Res. 1995;30:413-38.

29. Gualano B, Benatti FB, Batista-Ferreira JC, Franchini E, Brum PC, Lancha-Junior AH. Efeitos da suplementação de creatina no exercício intermitente de alta intensidade: divergências e recomendações metodológicas. Rev Bras Cineantropom Desempenho Hum. 2008;10(2):189-96.

30. Gualano B, Ugrinowitsch C, Novaes RB, Artioli GG, Shimizu MH, Seguro AC, et al. Effects of creatine supplementation on renal function: a randomized, double-blind, placebo-controlled clinical trial. Eur J Appl Physiol. 2008;103(1):33-40.

31. Gualano B, Ugrinowitsch $C$, Seguro AC, Lancha-Junior $\mathrm{AH}$. A suplementação de creatina prejudica a função renal? Rev Bras Med Esporte. 2008;14(1):68-73.

32. Hass CJ, Collins MA, Juncos JL. Resistance training with creatine monohydrate improves upper-body strength in patients with Parkinson disease: a randomized trial. Neurorehabil Neural Repair. 2007;21(2):107-15.

33. Hersch SM, Gevorkian S, Marder K, Moskowitz C, Feigin A, Cox M. Creatine in Huntington disease is safe, tolerable, bioavailable in brain and reduces serum 8OH2'dG. Neurology. 2006;66:250-2.

34. Horn M, Frantz S, Remkes H, Laser A, Urban B, Mettenleiter A, et al. Effects of chronic dietary creatine feeding on cardiac metabolism and on cretine content in heart, skeletal muscle, brain, liver and kidney. J Moll Cell Cardiol. 1998;30:277-84.

35. Hunter A. Monographs on biochemistry: creatine and creatinine, Londres: Longmans-Green; 1928.

36. Izquierdo M, Ibañez J, González-Badillo JJ, Gorostiaga EM. Effects of creatine supplementation on muscle power, endurance, and sprint performance. Med Sci Sports Exerc. 2002;34(2):332-43.

37. Kent-Braun JA, Sharma KR, Miller RG, Weiner MW. Post-exercise phophocreatine resynthesis is slowed in multiple sclerosis. Musc Nerv. 1994;17:835-41.

38. Khanna NK, Madan BR. Studies on the antiinflamatory activity of creatine. Arch Int Pharmac. 1978;231:340-50.

39. Kley RA, Tarnopolsky MA, Vorgerd M. Creatine treatment in muscle disorders: a meta-analysis of randomised controlled trials. J Neurol Neurosurg Psychiatry. 2008;79(4):366-7.

40. Kley RA, Vorgerd M, Tarnopolsky MA. Creatine for treating muscle disorders. Cochrane Database Syst Rev. 2007;24;(1):CD004760.

41. Koshy KM, Griswold E and Schneeberger EE. Interstitial nephritis in a patient taking creatine. $\mathrm{N}$ Engl J Med. 1999;340:814-5.

42. Kreider RB. Effects of creatine supplementation on performance and training adaptations. Mol Cell Biochem. 2003;244(1-2):89-94.

43. Kuehl K, Goldberg L, Elliot D. Renal insufficiency after creatine supplementation in a college football athlete. Med Sci Sports Exerc. 1998;30:S235.

44. Lauretani FC, Russo R, Bandinelli S, Bartali B, Cavazzini C, Di lorio A, et al. Age-associated changes in skeletal muscles and their effect on mobility: an operational diagnosis of sarcopenia. J Appl Physiol. 2003;95:1851-60.

45. Matthews RT, Yang L, Jenkins BG, Ferrante RJ, Rosen BR, Kaddurah-Daouk R, et al. Neuroprotective effects of creatine and cyclocreatine in animal models of Huntington's disease. J Neurosci. 1998;18:156-63.

46. McMorris T, Mielcarz G, Harris RC, Swain JP, Howard A. Creatine supplementation and cognitive performance in elderly individuals'. Neuropsychol Dev Cogn B Aging Neuropsychol Cogn. 2007;14(5):517-28.

47. Menezes LG, Sobreira C, Neder L, Rodrigues-Júnior AL, Martinez JA. Creatine supplementation attenuates corticosteroid-induced muscle wasting and impairment of exercise performance in rats. $\mathrm{J}$ Appl Physiol. 2007;102(2):698-703.

48. Murphy AJ, Watsford ML, Coutts AJ, Richards DA. Effects of creatine supplementation on aerobic power and cardiovascular structure and function. J Sci Med Sport. 2005;8(3):305-13.

49. Okudan N, Gokbel H. The effects of creatine supplementation on performance during the repeated bouts of supramaximal exercise. J Sports Med Phys Fitness. 2005;45(4):507-11.

50. Ostojic SM, Ahmetovic Z. Gastrointestinal distress after creatine supplementation in athletes: are side effects dose dependent? Res Sports Med. 2008;16(1):15-22.

51. Pearlman JP, Fielding RA. Creatine monohydrate as a therapeutic aid in muscular dystrophy. Nutr Rev. 2006;64:80-8.

52. Pereira B, Souza-Junior TP. Metabolismo celular e exercício físico - aspectos bioquímicos e nutricionais, São Paulo: Ed. Phorte; 2004.

53. Persky AM, Brazeau GA. Clinical pharmacology of the dietary supplement creatine monohydrate. Pharmacol Rev. 2001;53:161-76.

54. Pirola V, Pisani L, Teruzzi P. Evaluation of muscular trophicity in aged patients with femoral fractures treated with creatine phosphate and physiokinesiotherapy. Clin Ter. 1991;139:115-9.

55. Pline KA, Smith $C L$. The effect of creatine intake on renal function. Ann Pharmacother. 2005;39(6):1093-6.

56. Poortmans JR, Francaux M. Adverse effects of creatine supplementation: fact or fiction? Sports Med. 2000;30:155-70.

57. Pritchard NR, Kalra PA. Renal dysfunction 
accompanying oral creatine supplements. Lancet. 1998;351:1252-3.

58. Quinlivan RM, Beynon RJ. Pharmacological and nutritional treatment trials in McArdle disease. Acta Myol. 2007;26(1):58-60.

59. Rae C, Digney AL, McEwan SR, Battes TC. Oral creatine monohydrate supplementation improves brain performance: a double-blind, placebo-controlled, crossover trial. Proc Biol Scie. 2003;270:2147-50.

60. Reardon TF, Ruell PA, Fiatarone Singh MA, Thompson $\mathrm{CH}$, Rooney KB. Creatine supplementation does not enhance submaximal aerobic training adaptations in healthy young men and women. Eur J Appl Physiol. 2006;98(3):234-41.

61. Revai T, Sapi Z, Benedek S, Kovacs A, Kaszas I, Viranyi $M$, et al. Severe nephrotic syndrome in a young man taking anabolic steroid and creatine long term. Orv Hetil. 2003;144:2425-7.

62. Robinson TM, Sewell DA, Casey A, Steenge G, Greenhaff PL. Dietary creatine supplementation does not affect some haematological indices, or indices of muscle damage and hepatic and renal function. $\mathrm{Br} J$ Sports Med. 2000;34:284-8.

63. Sakellaris G, Kotsiou M, Tamiolaki M, Kalostos G, Tsapaki E, Spanaki M, et al. Prevention of complications related to traumatic brain injury in children and adolescents with creatine administration: an Open Label Randomized Pilot Study. J Trauma. 2006;61:322-9.

64. Satolli F, Marchesi G. Creatine phosphate in the rehabilitation of patients with muscular hypotrophy of the lower extremity. Curr Ther Res. 1989;46:67-73.

65. Schilling BK, Stone MH, Utter A, Kearney JT, Johnson $M$, Coglianese R, et al. Creatine supplementation and health variables: a retrospective study. Med Sci Sports Exerc. 2001;33:183-8.

66. Silva CA, Cancelliero KM. Efeito da suplementação oral com creatina no músculo esquelético de membro imobilizado de ratos Rev Bras Nutr Clin. 2006;21(1):17-22.

67. Sipilä I, Rapola J, Simell O, Vannas A. Suplementary creatine as a treatment for gyrate atrophy af the choroid and retina. NEJM. 1981;304:867-70.

68. Smith CA, Chetlin RD, Gutmann L, Yeater RA, Alway SE. Effects of exercise and creatine on myosin heavy chain isoform composition in patients with Charcot-Marie-tooth disease. Muscle Nerve. 2006;34(5):586-94.

69. Snow RJ, Murphy RM. Factors influencing creatine loading into human skeletal muscle. Exerc Sport Sci Rev. 2003;31(3):154-8.

70. Stromberger S, Bodamer OA, Stockler-ipsiroglu1 S. Clinical characteristics and diagnostic clues in inborn errors of creatine metabolism. J Inherit Metab Dis. 2003;26:299-308.

71. Sykut-Cegielska J, Gradowska W, Mercimek-Mahmu- toglu S, Stöckler-Ipsiroglu S. Biochemical and clinical characteristics of creatine deficiency syndromes. Acta Biochim Pol. 2004;51(4):875-82.

72. Syrotuik DG, Bell GJ. Acute creatine monohydrate supplementation: a descriptive physiological profile of responders vs. nonresponders. J Strength Cond Res. 2004;18(3):610-7.

73. Taes YEC, Delanghe JR, De Bacquer D, Langlois M, Stevens L, Geerolf I, et al. Creatine supplementation does not decrease total plasma homocysteine in chronic hemodialysis patients. Kidney Int. 2004;66:2422-8.

74. Taes YE, Delanghe JR, Wuyts $B$, van de Voorde $\mathrm{J}$, Lameire NH. Creatine supplementation does not affect kidney function in an animal model with pre-existing renal failure. Nephrol Dial Transplant. 2003;18:258-64.

75. Tarnopolsky MA. Potential benefits of creatine monohydrate supplementation in the elderly. Curr Opin Clin Nutr Metab Care. 2000;3(6):497-502.

76. Tarnopolsky MA, Mahoney DJ, Vajsar J, Rodriguez C, Doherty TJ, Roy BD, et al. Creatine monohydrate enhances strength and body composition in Duchenne muscular dystrophy. Neurology. 2004;62:1771-7.

77. Tarnopolsky MA, Martin J. Creatine monohydrate increases strength in patients with neuromuscular disease. Neurology. 1999;52:854-7.

78. Tarnopolsky MA, Roy BD, MacDonald JR. A randomized controlled trial of creatine monohydrate in patients with mitochondrial cytopathies. Musc Nerv. 1997;20:1502-9.

79. The American College of Sports Medicine. Roundtable: the physiological and health effects of oral creatine supplementation. Med Sci Sports Exerc. 2000;32:706-17.

80. Thorsteinsdottir B, Grande JP, Garovic VD. Acute renal failure in a young weight lifter taking multiple food supplements, including creatine monohydrate. J Ren Nutr. 2006;16:341-5

81. van Loon LJ, Oosterlaar AM, Hartgens F, Hesselink MK, Snow RJ, Wagenmakers AJ. Effects of creatine loading and prolonged creatine supplementation on body composition, fuel selection, sprint and endurance performance in humans. Clin Sci (Lond). 2003;104(2):153-62.

82. Vannas-Solunen K, Sipilä I, Vannas A, Simell O, Rapola J. Gyrate atrophy of the choroid and retina. Ophtalmology. 1985;92:1719-27.

83. Vieira RP, França RF, Carvalho CRF, Dolhnikoff M, Ribeiro W, Martins RAB. Efeitos da Suplementação Oral com Creatina sobre o Metabolismo e a Morfologia Hepática em Ratos. Rev Bras Med Esporte 2008; 14(1): 38-41.

84. Volek JS, Ratamess NA, Rubin MR, Gómez AL, French DN, McGuigan MM, et al. The effects of creatine supplementation on muscular performance 
Câmara LC, Dias RMR. Suplementação de creatina: efeitos ergogênicos e terapêuticos.

and body composition responses to short-term resistance training overreaching. Eur J Appl Physiol. 2004;91(5-6):628-37.

85. Williams MH, Kreider RB, Branch JD. Creatina. 2a ed. São Paulo: Manole; 1999.

86. Williams $\mathrm{MH}$. Nutritional aspects of human physical and athletic performance. Springfield, Illinois: Charles C Thomas; 1985.

87. Wittingham TS, Lipton P. Cerebral synaptic transmition during anoxia is protected by creatine. $\mathrm{J}$ Neurochem.
1981;37:1618-21.

88. Wyss M, Kaddurah-Daouk R. Creatine and creatinine metabolism. Physiol Rev. 2000;80:1107-213.

89. Wyss M, Schulze A. Health implications of creatine: can oral creatine supplementation protect against neurological and atherosclerotic disease? Neuroscience. 2002;112(2):243-60.

90. Yoshizumi WM, Tsourounis C. Effects of creatine supplementation on renal function. J Herb Pharmacother. 2004;4(1):1-7.

Recebido para publicação: 24/04/2009

Aceito para publicação: 11/09/2009 